\title{
On the Computation of Conversational Implicatures
}

\author{
Uli Sauerland \\ Tübingen University
}

\section{Introduction}

\subsection{A Starting Point}

Since the work of Horn (1972) and Gazdar (1979), conversational implicatures triggered by scalar expressions are generally regarded as fairly well understood, at least at a descriptive level (see e.g. the textbooks by Levinson (1983:132-36) and Gamut (1991:204-209)). The class of phenomena this optimism is based on are exemplified in (1) and (2). For these cases, it's generally agreed that the second sentence is an implicature of the first.

(1) Kai had peas or broccoli last night.

$\sim$ Kai didn't have peas and broccoli last night.

(2) Kai had some of the peas last night.

$\sim$ Kai didn't have all of the peas last night.

Gazdar (1979:57-61) proposes a precise mechanism for the computation of such scalar implicatures (similar descriptions have been given by Horn 1989:214, Atlas and Levinson 1981:33, Levinson 2000:76). It relies on the notion of a quantitative scale that Horn (1972) introduced. Gazdar's mechanism applies only to sentences $\phi$ where an expression from a quantitative scale $\alpha$ appears not in the scope of any logical operator. To such a sentence it assigns the set of expressions of the form $\mathrm{K} \neg \psi$, where $\psi$ is derived from $\phi$ by replacing one occurrence of $\alpha$, that's not in the scope of any logical operator, with $\alpha^{\prime}$ and there's a scale $Q$ on which $\alpha^{\prime}$ is the word following $\alpha . \mathrm{K}$ here is the epistemic certainty operator of Hintikka (1962), so the implicatures of $\phi$ can each be paraphrased as Certainly not $\psi$ where $\psi$ is related to $\phi$ by a certain replacement operation. ${ }^{1}$

Consider the examples in (1) and (2) for an illustration. The quantitative scales required in the account of (1) and (2) are 〈or, and and 〈some, all $\rangle$. In each of the examples (1) and (2), there's only one possible replacement to consider, so there's also only one implicature in each example. The implicature assigned to (1) is then $\mathrm{K} \neg$ Kai had peas and broccoli last night, and the one assigned to (2) is $\mathrm{K} \neg$ Kai had all of the peas last night. Since, at least roughly, anything that's certain is assertable and vice versa, the implicatures predicted by Gazdar's mechanism for (1) and (2) correspond to the indicated intuitions.

\subsection{Limitations of Gazdar's Mechanism}

Gazdar's procedure is very limited since it only applies to cases that unlike (1) and (2) contain scalar terms in the scope of a logical operator. This would seem 
to predict that scalar terms in the scope of a logical operator never lead to any implicatures. As other people have noticed this before, this prediction is incorrect. ${ }^{2}$

While the class of problems associated with negation is of ten discussed, the more serious problems actually arise in examples without negation and downward entailing operators. In particular, there is a problem extending Gazdar's procedure to cases with disjunction and another operator in its scope. This problem in its full generality was brought to my attention by Gennaro Chierchia and Bernhard Schwarz. ${ }^{3}$ Consider example (3), which looks like an innocent combination of (1) and (2).

(3) Kai had some of the peas or the broccoli last night.

As we'll see, (3) is actually a serious challenge for the theory of implicatures. Consider first what Gazdar predicts: Since Gazdar's mechanism can only apply to occurrences of scalar terms not in the scope of any other logical operator, it only applies to "or" in (3). The occurrence of "some" in (3) is in the scope of "or" and therefore not in the domain of Gazdar's mechanism. Therefore, only one implicature is predicted for (3), namely the one given in (4). ${ }^{4}$

(4) (3) $\leadsto$ Kai didn't have some of the peas and the broccoli last night.

While this implicature is intuitively correct, both (3) and (4) would be logically true in a situation where Kai ate all of the peas and no broccoli last night, and therefore should a felicitous utterance in such a situation. But, this doesn't seem to be right. If you were to utter (3), while I knew for a fact that Kai had all the peas last night, I would think you're wrong. Hence, in such a scenario, I would use (5a) in my reply to you, rather than $(5 b)$.

(5) a. No, he had all of the peas last night.

b. \#Yes, he had all of the peas last night.

So, there seems to be at least one other implicature that's drawn from (3). For example, if (6) could be drawn as an implicature from (3) this would capture the intuition just observed.

(6) (3) $\leadsto$ Kai didn't have all of the peas last night.

But, how to arrive at something like (6)?

\subsection{Contributions of this Paper}

As Chierchia (2001) argues, it isn't obvious how to extend something like Gazdar's procedure to cases where a scalar term appears in the scope of another logical operator. Therefore, Chierchia dismisses the entire picture of Grice (1978) and develops one of his own.

My goal is to show that such a radical break is unwarranted. I show that the disjunction problem can be solved within Grice's framework. 
This paper makes two contributions that are of a more technical nature. On the one hand, I show that in general the implicatures of sentences containing more than one scalar expression are computed by forming something akin to the crossproduct of the two scales. On the other hand, I'll argue that the scale of disjunction is not just 〈or, and , but rather is a set that is only partially ordered by entailment and contains the items "A or B", "A", "B", and "A and B". Furthermore, I provide some fairly direct empirical evidence for the claim of e.g. Soames (1982) and Horn (1989) that implicatures are epistemically modalized.

I should mention that there's one important question that I have nothing to say about here: Namely, the question where quantitative scales come from. I'll here simple take quantitative scales for granted and use them to account for the implicatures of sentences. See Hirschberg (1985), Matsumoto (1995), and von Fintel and Heim (2000) for some interesting suggestions relating to this question.

\section{Crossing Scales}

\subsection{A Small Improvement}

What implicatures are drawn from examples with a scalar term in the scope of a logical operator? One initial problem to overcome is to deal with scalar terms in the scope of negation. Consider example (7) from Gazdar (1979). ${ }^{5}$ Here, the simpleminded application of Gazdar's procedure would, by replacing some with all and negating the result (where then the double negations cancel each other out), arrive at the result in the second line of (7).

(7) It is not the case that Paul ate some of the eggs.

$\not \nrightarrow$ Paul ate all the eggs. (Gazdar 1979:56)

But clearly, this result is incorrect for (7) - the putative implicature contradicts the assertion. The root of the problem is that negation reverses scales (Horn 1972:33, Atlas and Levinson 1981:33). Replacing some with all, when it's not in the scope of negation, often yields a stronger proposition, and if that's the case Gazdar's procedure yields the right result. But when some occurs in the scope of negation as in (7), replacing it with all yields a weaker claim. That's why Gazdar had to block his procedure from applying in this case.

Gazdar's solution to the negation problem-blocking implicature computation from applying to any scalar term in the scope of a logical operator is stipulative, and doesn't predict the implicature reversal actually observed (Atlas and Levinson 1981:33 and others). A variant of Gazdar's mechanism that avoids the problem with negation in a better way is based on the idea to divorce the replacement of a scalar term by an alternative from the computation of whether the result of the replacement lead to a stronger claim. While this intuition seems to have been around since Horn (1972), I'm not aware of technical implementation of this idea. I propose the following terminology to capture the sentences derivable by replacements of scalar 
terms with terms from the same scale: ${ }^{6}$

(8) A sentence $\psi$ is one-step scalar alternative of $\phi$ if the following two conditions hold:

a. $\phi \neq \psi$

b. there are scalar expressions $\alpha$ and $\alpha^{\prime}$ which both occur on the same scale $C$ such that $\psi$ is the result of replacing one occurrence of $\alpha$ in $\phi$ with $\alpha^{\prime}$.

(9) A sentence $\psi$ is a scalar alternative of $\phi$ if there is a sequence $\left(\phi_{0}, \ldots, \phi_{n}\right)$ with $n \geq 0$ and $\phi_{0}=\phi$ and $\phi_{n}=\psi$ such that for all $i$ with $i \geq 1$ and $i \leq n \phi_{i}$ is a one-step scalar alternative of $\phi_{i-1}$.

The proposal is then that a scalar alternative of an asserted sentence leads to an implicature only if the scalar alternative is stronger than the assertion. This is given in (10).

(10) $\neg \alpha^{\prime}$ is an implicature of $\alpha$ if the following three hold:

a. $\alpha^{\prime}$ is a scalar alternative of $\alpha$

b. $\alpha^{\prime}$ entails $\alpha$

c. $\alpha$ doesn't entail $\alpha^{\prime}$

Proposal (10) solves the negation problem: While an implicature is blocked in Gazdar's example (7), there's an implicature in the reverse direction in (11).

(11) It's not the case that Paul ate all of the eggs.

$\sim$ Paul ate some of the eggs.

\subsection{Crossing Scales}

The proposal just developed makes a prediction for the implicatures of sentences that contain more than one scalar term. Abstractly, we can view a sentence with two occurrences of a scalar term as $\phi(X, Y)$, where $X$ is an element of the quantitative scale $Q_{X}$ and $Y$ an element of the scale $Q_{Y}$. The set of scalar alternatives of $\phi(X, Y)$ is then isomorphic to the cross product of the two scales $Q$ and $Q^{\prime}$. This is apparent from the fact that (12) holds:

(12) ScalAlt $(\phi(X, Y))=\left\{\phi\left(X^{\prime}, Y^{\prime}\right) \mid X^{\prime}\right.$ an element of $Q_{X}, Y^{\prime}$ an element of $\left.Q_{Y}\right\}$

The predicted implicatures are then all those sentences $\phi\left(X^{\prime}, Y^{\prime}\right)$ that entail $\phi(X, Y)$. To a large extent this prediction is borne out, but as we'll see there are a few remaining problems (including the recalcitrant (3) from above).

Consider first examples where neither of the two scalar expressions is in the scope of each other, nor in the the scope of any other logical operator. For such cases, Gazdar's procedure actually makes the same prediction as the system developed above. It's not easy to come up with examples that satisfy this restriction, but (13) and (14) can I think be argued to do so. For (14), though, this is only the case for the cumulative reading, which is indicated by the **-operator (Beck and 
Sauerland 2001 and references therein) and even that might be questionable. The predicted implicatures are shown below these two examples.

(13) The father of some of the boys knew some of the answers.

$\leadsto \neg$ (the father of every boy knew some of the answers)

$\leadsto \neg$ (the father of some of the boys knew all of the answers)

$\sim \neg$ (the father of all of the boys knew all of the answers)

(14) 600 Dutch firms **have 5000 American computers. (Scha 1984:146)

$\leadsto \neg$ (601 Dutch firms $* *$ have 5000 computers)

$\leadsto \neg(600$ Dutch firms **have 5001 computers)

$\sim$... (all other implicatures are entailed by the previous two)

Are these implicatures actually correct? While my intuition looking at this examples is a little unclear, if I consider them as answers to a relevant question, I think they are in accord with the prediction. ${ }^{7}$ For (13) such a context would be provided by the question Who knew how many of the answers?, and for (14) by How many firms have how many computers?. It helps with the judgement on (14) to slightly change the numbers in the example; for instance, to 599 and 4999.

A further argument that (13) has indeed the predicted implicatures is provided by the data in (15).

(15) a. The fathers of some of the boys knew some of the answers, and possibly even the father of all of the boys knew some of the answers.

b. The fathers of some of the boys knew some of the answers, and possibly the father of some of the children knew even all of the answers.

Horn (1972:30) argues that suspender clauses (i.e. clauses that cancel an implicature) like those initiated by and possibly even are only acceptable, if they express an actual implicature. Therefore, the acceptability of the data in (16) argues that (14) indeed has the two first implicatures given in (14).

Now consider sentences where there's a scopal relation. Specifically consider (16) and (17), which fall outside of the scope of Gazdar's mechanism, since here both occurrences of scalar terms other than and are in the scope of and in both examples. The implicatures predicted by the proposal currently under investigation are shown below the assertion in (16) and (17).

(16) John read A or B and watched C or D.

$\leadsto \neg($ John read A and B and watched C or D)

$\leadsto \neg($ John read A or B and watched C and D)

$\leadsto \neg$ (John didn't read A and B and watched C and D)

(17) Kai ate some of the strawberries and Hannes ate some of the carrots.

$\sim \neg$ (Kai ate all of the strawberries and Hannes ate some of the carrots)

$\leadsto \neg$ (Kai ate some of the strawberries and Hannes ate all of the carrots)

$\leadsto \neg$ (Kai ate all of the strawberries and Hannes ate all of the carrots) 
For both (16) and (17), the predicted implicatures shown seem to be correct. To make the judgement easier, consider that the conjunction of the assertion and the implicatures of (17) entail the two statements in (18). ${ }^{8}$

(18) a. Kai didn't eat all of the strawberries.

b. Hannes didn't eat all of the carrots.

Examples (16) and (17) illustrate scalar expressions in the scope of negation. I don't have examples with two scalar expressions in the scope of negation, but not in the scope of each other, since I found these too difficult to form a judgment on. But, consider (19), which seems to behave as predicted.

(19) It's not the case that every child knows every parent.

$\sim$ Some child knows every parent.

$\leadsto$ Every child knows some parent.

$\leadsto$ Some child knows some parent.

Finally consider examples where one scalar expression occurs in the scope of another. This class of examples will be most relevant for the remainder of this paper. Consider first the examples (20) and (21). Again I show the predicted implicatures below each of the examples.

(20) Winnie is allowed to smoke three cigarettes today.

$\leadsto$ Winnie isn't required to smoke three cigarettes today.

$\leadsto$ Winnie isn't allowed to smoke four cigarettes today.

$\sim$... (all other implicatures are entailed by the previous two)

(21) Some of the children found some of their presents.

$\sim$ Not all of the children found some of their presents.

$\leadsto$ Some of the children didn't find all of their presents.

$\leadsto \neg$ (All of the children found all of their presents)

Again, the predicted implicatures are intuitively correct in both of (20) and (21). As the following section shows, though, there are certain systematic exceptions to the prediction made amongst examples with a scalar expression below another.

\subsection{Remaining Problems}

At present, I'm aware of two cases where the account developed in 2.1 makes empirically wrong predictions. These are, on the one hand, examples where a scalar term occurs in the scope of disjunction, which were already mentioned in (3) above. On the other hand, cases where a scalar term occurs in the scope of an epistemic modal turn out to be problematic.

Consider now the first class of empirical problems: Scalar expressions in the scope of disjunction. In (22), example (3) is repeated with the two implicatures that were observed above.

(22) Kai had some of the peas or the broccoli last night. 
$\leadsto$ Kai didn't have some of the peas and the broccoli last night.

$\leadsto$ Kai didn't have all of the peas last night.

To see what the proposal under discussion predicts, observe the set of scalar alternatives of (22) in (23).

(23) $\operatorname{ScalAlt}((22))=\{a, b, c\}$

a. Kai had all of the peas or the broccoli last night.

b. Kai had some of the peas and the broccoli last night.

c. Kai had all of the peas and the broccoli last night.

Scalar implicatures arise from those scalar alternatives that entail (22). Since all three of (23a), (23b) and (23c) entail (22), three implicatures are predicted. While (23b) and (23c) don't cause any problems, (23a) predicts that (24) should be an implicature of (23).

(24) $\chi_{\rightarrow} \neg$ (Kai had all of the peas or the broccoli last night.)

This result, however, is wrong (Chierchia 2001:6). (24) entails that Kai didn't have the broccoli last night. But, clearly (22) doesn't implicate that Kai didn't have the broccoli last night. ${ }^{9}$

As I mentioned above the problem occurs more generally with scalar expressions in the scope of disjunction. Consider Simons's (1998:94) discussion of (25) (possibly the point goes back to McCawley (1993), see footnote 3).

(25) $\mathrm{A}$ or B or C

$\sim \operatorname{not}(\mathrm{A}$ and $\mathrm{B})$

$\sim \operatorname{not}(\mathrm{A}$ and $\mathrm{C})$

$\sim \operatorname{not}(\mathrm{B}$ and $\mathrm{C})$

Simons observes that the implicatures shown in (25) are the ones the sentence actually has. These are equivalent to the claim that only one out of $\mathrm{A}, \mathrm{B}$ and $\mathrm{C}$ is true. Compare this to the predictions of the current proposal shown in (26).

(26) Predictions for (25)

a. $\leadsto \neg(\mathrm{A}$ and $(\mathrm{B}$ or $\mathrm{C}))$

b. $\quad \chi_{\rightarrow} \neg(\mathrm{A}$ or $(\mathrm{B}$ and $\mathrm{C}))$

Since (26b) entails $\neg \mathrm{A},(26 \mathrm{~b})$ can't be an implicature of (25), which would be an acceptable assertion in case the speaker knew that $\mathrm{A}$ is false.

The other family of problems for the proposal in section 2.1 are scalar terms under epistemic modals. The problem is illustrated by the minimal pairs comparing epistemic might with a different modal. ${ }^{10}$

(27) a. Winnie might smoke three cigarettes today.

b. Winnie is allowed to smoke three cigarettes today. $(=(20))$

(28) a. Kai possibly ate seven grapes. 
b. Kai is capable of eating seven grapes.

The pairs in (27) and (28) differ in whether an implicature based on the scalar alternatives derivable by strengthening the scalar term under the modal is observed. The felicity of the responses in (29) for (27) and in (30) for (28) brings out the difference.

(29) a. \#No, Winnie possibly smokes four cigarettes today.

b. No, Winnie is allowed to smoke four cigarettes today.

(30) a. \#No, Kai possibly ate eight grapes.

b. No, Kai is capabale of to eating eight grapes.

With epistemic might, there's no implicature of the form $\neg$ might [a stronger alternative of the scope of might]. And possibly behave analogous. However, such an implicature is predicted for might in just the same way as observed with other existential modals like allowed to in (20) and in (28b) above, and in a similar fashion for possibly. This problem, I at present don't have a solution for. ${ }^{11}$

\section{Disjunction and the Epistemic Step}

\subsection{The Scale of Disjunction}

In this section, I consider the cases with disjunction that has a scalar term in its scope. We saw above that the proposal developed in section 2.1 didn't predict the second implicature in example (31) (repeated from (3)).

(31) Kai had some of the peas or the broccoli last night.

$\leadsto$ Kai didn't have some of the peas and the broccoli last night.

$\leadsto$ Kai didn't have all of the peas last night.

Which of the assumptions going into the proposal 2.1 could be changed to predict this implicature. A straightforward way of adding the implicature to the set of implicatures predicted by the proposal is to assume the more elaborate scale for disjunction in (32).

(32) The Scale of Disjunction

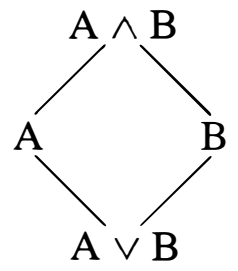

The diamond shape expresses the opinion that, in contrast to other cases of quantitative scales, the scale of disjunction is a set that is only partially ordered by entailment. 
If the scale of a disjunction is indeed the one shown in (32), the set of scalar alternatives of (31) includes the sentence (33).

(33) Kai had all the peas last night.

From (33), it immediately follows that the second implicature in (31) is predicted to be an implicature of (31).

However, the assumption that (32) is the scale of disjunction makes a number of undesirable predictions. For one, it predicts that for any assertion $A$ the following should hold: for any proposition $B$, since $A$ is on the scale in (32), A $\wedge \mathrm{B}$ should be a scalar alternative of $\mathrm{A}$. But, since $\mathrm{A} \wedge \mathrm{B}$ entails $\mathrm{A}, \neg(\mathrm{A} \wedge \mathrm{B})$ should be an implicature of $\mathrm{A}$ for any $\mathrm{B}$. From this implicature and the assertion A, however, $\neg \mathrm{B}$ follows. This prediction is clearly undesired: Asserting "It rains" implicates neither "John sneezed" nor "John didn't sneeze", though both of this implicatures are predicted at this point. One way to rule out these particular prediction would be to say that, since, assuming the truth of the assertion, they contradict each other, they cancel each other out. However, clearly any cognitve mechanism actually computing implicatures must know in advance that these infinitely many potential implicatures don't arise. Therefore, it might be preferable to block such implicatures in a more direct manner. One option in this direction would be to assume that the scale of disjunction actually consists only of binary proposition operators, but that there are lexical entries for two such operators $\mathrm{L}$ and $\mathrm{R}$ defined as following. ${ }^{12}$

(34) for any two propositions $\mathrm{A}, \mathrm{B}: \mathrm{A} \mathrm{L} \mathrm{B}=\mathrm{A}$ and $\mathrm{A} \mathrm{R} B=\mathrm{B}$

Since now A is not actually an element of a scale, the problem noted above doesn't arise. Evidently though this solution is more of a technical trick, than a real solution for the problem noted above. Nevertheless, I'll assume it for now.

A second problem has a more fruitful effect, I believe. For a statement of the form $\mathrm{A} \vee \mathrm{B}$ the scale in (32) predicts the implicatures $\neg \mathrm{A}$ and $\neg \mathrm{B}$ since both $\mathrm{A}$ and $B$ are scalar alternatives of $A \vee B$. But, these two predicted implicatures don't actually seem to be observed. At least in example (35), it's odd to cancel one of these putative implicatures.

(35)\#It's raining or Kai is peeing a lot and possibly it's even raining.

I believe that we nevertheless want to continue to assume something like the scale in (32) for disjunction. The problem observed here can, as I'll show now, be related to an old, controversial issue in the computation of implicatures-the epistemic status of the implicature.

\subsection{The Epistemic Status of Implicatures}

My starting point in this investigation has been the mechanism Gazdar (1979) proposes to predict the implicatures of a sentence $\phi$. Soames (1982:521) and Horn (1989:543, n5), criticize one aspect of Gazdar's mechanism: namely, the epistemic 
commitment assigned to the implicatures by Gazdar's proposal. While both Soames and Horn agree that implicatures of $\phi$ arise from certain stronger statements $\psi$, they argue that it only follows from Gricean maxims of conversation that $\psi$ is uncertain, rather than that $\psi$ is certainly false as Gazdar claims. ${ }^{13}$ Following Gazdar, I'll employ the K-operator to express epistemic certainty, and the P-operator for epistemic possibility (Hintikka 1962). Using this terminology, Soames and Horn's claim is that implicatures are initially $\neg \mathrm{K} \psi$ rather than $\mathrm{K} \neg \psi$. Furthermore Soames and Horn point out that Gazdar's K $\neg \psi$ follows from their initial implicature if it's assumed that e.g. $\mathrm{K} \psi \vee \mathrm{K} \neg \psi$ holds, and that in fact we usually seem to make some such additional assumption in conversation. This then explains the fact that Gazdar's proposal made the right empirical prediction in many cases.

The proposal in section 2.1 adopted the assumption from Gazdar that the procedure directly arrives at a set of epistemically certain implicatures of the form $\mathrm{K} \neg \psi$. Therefore, the criticism mentioned applies to this proposal in the same way. In the analysis I develop for the disjunction problem in the following, the point Soames and Horn raise is an important part of the solution. I claim that the distinction between the "uncertain $\psi$ " implicatures that follow from Gricean maxim's of conversation, and the "certain not $\psi$ " implicatures Gazdar predicts is important for the explanation of the implicatures of disjunction. Therefore, I introduce a terminological distinction between these two types of implicatures: I'll call the Soames/Horn type implicatures primary implicatures, and the Gazdar type implicatures secondary implicatures. An set of all implicatures predicted by this analysis is then the unions set of the sets of primary or a secondary implicatures.

(36) If $\psi \in \operatorname{ScalAlt}(\phi)$ and $\psi \Rightarrow \phi$ and not $\phi \Rightarrow \psi$, then $\neg \mathrm{K} \psi$ is a primary implicature of $\phi$.

(37) If $\neg \mathrm{K} \psi$ is a primary implicature of $\phi$ and $\mathrm{K} \neg \psi$ is consistent with the conjunction of $\phi$ and all primary implicatures of $\phi$, then $\mathrm{K} \neg \psi$ is a secondary implicature of $\phi$.

The condition in (37) rules out the case in which a secondary implicature contradicts the conjunction of the primary implicatures and the assertion. This assumption introduces a hierarchy amongst the implicatures, and one must ask why it's not possible to cancel the primary implicature or the assertion, rather than the secondary implicature if there is a conflict between the two. However, this hierarchy follows if we consider the sets of assumptions that go into the reasoning that one of these is true. To reason that the assertion is true, one only needs to appeal to the assumption that the speaker making an assertion is obeying one of Grice's maxims, namely "Tell the truth". To reason that the primary implicatures arise, one needs the assumption that the speaker making an assertion is obeying all of Grice's maxims. ${ }^{14}$ Finally, to reason for a secondary implicature $\mathrm{K} \neg \psi$ one has to assume that the speaker obeys all the Gricean maxims to derive $\neg \mathrm{K} \psi$ and furthermore that $\mathrm{K} \psi \vee \mathrm{K} \neg \psi$ or some stronger assumption is justified.

The distinction between primary and secondary implicatures now leads to a new prediction when combined with the scale for disjunction in (32). Consider 
a abstract statement of the form $A \vee B$. From the scalar alternatives in (32), the primary implicatures in (38) follow straightforwardly.
a. $\neg \mathrm{K} \mathrm{A}$
b. $\neg \mathrm{K} \mathrm{B}$
c. $\neg \mathrm{K}(\mathrm{A} \wedge \mathrm{B})$

The implicatures (38a) and (38b) are already observed by Gazdar (1979:50,(41)). Therefore, the result that we predict these implicatures is empirically correct. Since, (38c) is entailed by (38a) or (38b) it's also empirically confirmed. Gazdar accounts for the implicatures (38a) and (38b) as clausal quantity implicatures (page 59). It does, however, seem desirable to eliminate this concept which Gazdar only makes use of to account for two examples: (40) and (41) on page 50. I believe this to be possible on the basis of the distinction between primary and secondary implicatures developed here. ${ }^{15}$

Which of the primary implicatures in (38) gives rise to secondary implicatures? To answer this question, we need to decide which of the the potential secondary implicatures are consistent with the conjunction of all primary implicatures and the assertion. Consider first the secondary implicature arising form (38a), namely $\mathrm{K} \neg \mathrm{A}$. This secondary implicature is blocked because it follows from the assertion $\mathrm{K}(\mathrm{A} \vee \mathrm{B})$ and the primary implicature (38b), that A must possibly be true. The secondary implicature $\mathrm{K} \neg \mathrm{B}$, which could arise from (38b), is blocked in a similar way. Finally, consider the secondary implicature $\mathrm{K} \neg(\mathrm{A} \wedge \mathrm{B})$. This implicature is consistent with the assertion and the primary implicatures, and therefore predicted to actually arise. This prediction is actually borne out, since this implicature is in fact the exclusiveness implicature of disjunction illustrated by (1) at the beginning. This result is summarized in (39):

(39) secondary implicatures of $\mathrm{A} \vee \mathrm{B}$ :
a. $\quad \not \rightarrow \mathrm{K} \neg \mathrm{A}$ (blocked)
b. $\not \triangleleft \mathrm{K} \neg \mathrm{B}$ (blocked)
c. $\sim \mathrm{K} \neg(\mathrm{A} \wedge \mathrm{B})($ Exclusiveness $)$

\subsection{Scalar Terms in the Scope of Disjunction}

Now, we're ready to address example (3) and the general class of problematic examples it exhibits: examples with a scalar term in the scope of disjunction. I repeat example (3) with its implicatures in (40).

(40) Kai had some of the peas or the broccoli last night.

$\leadsto$ Kai didn't have some of the peas and the broccoli last night.

$\leadsto$ Kai didn't have all of the peas last night.

Consider first the list of primary implicatures predicted by the approach of the previous section. 
(41) a. $\neg \mathrm{K}(\mathrm{Kai}$ had some of the peas last night)

b. $\neg \mathrm{K}(\mathrm{Kai}$ had the broccoli last night)

c. $\neg \mathrm{K}$ (Kai had some of the peas and the broccoli last night)

d. $\neg \mathrm{K}(\mathrm{Kai}$ had all of the peas last night)

e. $\neg \mathrm{K}(\mathrm{Kai}$ had all of the peas or the broccoli last night)

f. $\neg \mathrm{K}(\mathrm{Kai}$ had all of the peas and the broccoli last night)

Again, all of these primary implicatures seem unproblematic.

Consider now the secondary implicatures that potentially arise from (41). As we've already seen by means of the abstract consideration of $A \vee B$ in (38) above the secondary implicatures from (41a) and (41b) are blocked, while (41c) gives rise to a secondary implicature.

Now consider $\mathrm{K} \neg$ (Kai had all of the peas last night), which arise from (41d). This is actually consistent with the the assertion shown in (40) and all the primary implicatures shown in (41). This is demonstrated by the fact that there are scenarios where the assertion, all primary implicatures, and $\mathrm{K} \neg$ (Kai had all of the peas last night) holds. Consider the following: I wasn't present for Kai's dinner, but I conclude from circumstantial evidence that he must've had peas or broccoli last night. Furthermore, I saw some peas in the fridge, but I don't remember whether there were more peas. So, I'm sure that Kai didn't eat all the peas, but I don't know whether any of them disappeared. I didn't investigate whether there's broccoli in our fridge. In this scenario, I'm certain that Kai had some of the peas or the broccoli (the assertion). I'm not certain that he had some of the peas ((41a)), or even all of the peas $((41 \mathrm{~d}))$, or that he had the broccoli $((41 \mathrm{~b}))$. To verify the other three primary implicatures, recall the following equivalences that generally hold: $\neg \mathrm{K} \phi$ is equivalent to $\mathrm{P} \neg \phi, \neg(\mathrm{A}$ or $\mathrm{B})$ is equivalent to $\neg \mathrm{A}$ and $\neg \mathrm{B}$, and $\mathrm{P}(\mathrm{A}$ or $\mathrm{B})$ is equivalent to $\mathrm{PA}$ or $\mathrm{PB}$. Hence, the truth of (41c) can be seen by considering that it's possible that Kai didn't have some of the peas. The equivalencies in (42) follow:

(42) (41c) $\Leftrightarrow P \neg($ Kai had some of the peas last night) or $\mathrm{P} \neg$ (Kai had the broccoli last night)

(41e) $\Leftrightarrow P$ (Kai didn't have all of the peas last night and didn't have the broccoli last night)

(41f) $\Leftrightarrow P \neg($ Kai had all of the peas last night) or $\mathrm{P} \neg($ Kai had the broccoli last night)

Now, it's easy to see that (41c) and (41f) are entailments of (41b). That (41e) is true in the scenario given can be verified by considering that everything said there is compatible with Kai having only eaten some of the peas and nothing else. Finally, in the scenario it's certain that Kai didn't eat all the peas, hence the putative secondary implicature is also fulfilled. Hence, this secondary implicature is actually predicted.

Now consider the secondary implicature arising from (41e): $\mathrm{K} \neg(\mathrm{Kai}$ had all of the peas or the broccoli last night). It follows from the assertion and the primary implicature (41a) that $\mathrm{P}$ (Kai had the broccoli last night) must hold. Since this 
is inconsistent with the secondary implicature arising from (41e), this secondary implicature must be blocked.

Finally, the secondary implicature from (41f) $-K \neg$ (Kai had all of the peas and the broccoli last night) - is entailed by the secondary implicature contributed by (41d). Hence, it isn't blocked, but also doesn't provide any additional information. In sum, we have therefore shown that the two secondary implicatures in (42) are predicted by the approach developed in the previous section.

(43) $\sim \mathrm{K} \neg$ (Kai had some of the peas and the broccoli last night)

$\sim \mathrm{K} \neg$ (Kai had all of the peas last night)

These are the two implicatures that the example (3) was argued to have. We have now accounted for the example (3). I leave it up to the reader to verify that the proposal extends to the other cases of scalar terms in the scope of disjunction noted in section 2.3 above.

\section{Conclusion}

The mechanism for the generation of scalar implicatures discussed in this paper has allowed us to predict the right implicatures for examples like (44) (repeated from (3)). I argued that, in general, implicatures can arise from scalar expressions embedded under logical operators, but that one must be careful when considering examples like (44) where a scalar term occurs within the scope of disjunction.

(44) Kai had some of the peas or the broccoli last night.

$\sim$ Kai didn't have some of the peas and the broccoli last night.

$\sim$ Kai didn't have all of the peas last night.

I have shown that a simple picture of how scalar implicatures can be uniformly computed as the negation of all the logically strictly stronger scalar alternatives according to the definitons in section 2.1.

I then showed one class of cases that still remained problematic; namely, cases of a scalar term embedded under disjunction. I showed that an account of such cases is possible based on two assumptions. The first assumption is that the scale of a disjunction $A \vee B$ isn't simply $A \wedge B$, but also includes $A$ and $B$. This scale is introduced in (32). The second assumption was the adoption of a two step approach to implicatures as characterized by different epistemic quality. This result is presented in section 3.3.

\section{Endnotes}

The ideas in this paper are a summary of my reactions to two separate presentations by Gennaro Chierchia and Bernhard Schwarz at Tübingen University in October 2000 and subsequent discussions with both of the authors. I'm very grateful to both 
of them for their insights and their willingness to discuss these issues with me. Kai von Fintel also deserves special thanks for making it clearer to me what I was saying and how it relates to what other people have said. An earlier version of this paper was presented at the Workshop on Formal Pragmatics at the Zentrum für allgemeine Sprachwissenschaft at Berlin. I also thank Manfred Krifka, Laurence Horn, Irene Heim, Ede Zimmermann, Robert van Rooy, Winnie Lechner, Fritz Hamm, Kazuko Yatsushiro, Martin Hackl, and Elena Guerzoni for useful comments along the way. 1. Gazdar (1979) distinguishes in his terminology between the potential implicatures of a sentence and its actual implicatures. The results of his mechanism are actual implicatures of a particular sentence only if they're not cancelled by one of Gazdar's implicature cancellation conventions. My use of terminology in the text therefore is sloppy, but I believe sufficiently clear for my purposes.

2. In particular, Hirschberg (1985:72-74) suggests that Gazdar actually could do with a much more lenient restriction. Namely, she proposes that only scalar terms in the scope of negation are excluded from Gazdar's mechanism. However, as will become clear from the following discussion and is shown in footnote 4 , Hirschberg's suggestion has a number of problems.

3. A special case of this puzzle, namely the case of disjunction below another disjunction, seems to be aimed at by McCawley (1993:324) in an exercise and is discussed by Simons (1998:88-96) (The first edition of McCawley's book (from 1981) might state the problem more directly, but I have not been able to access this reference.)

4. Hirschberg's (1985) suggestion (see footnote 2) predicts (i) to be an implicature of (3) (her example (75c) on page 73 is analogous).

(i) $\leadsto$ It's not the case that Kai had all of the peas or the broccoli last night.

But, (i) is too strong since it entails that Kai didn't have the broccoli.

5. To my knowledge examples like (45) are the only reason Gazdar restricts his mechanism to occurrences of scalar terms occurring in the scope of no other logical operator.

6. Note that from this point on quantitative scales themselves don't need to be represented as ordered lists anymore. It would be sufficient to represent them as plain sets. I'll stick to the tradition though.

7. Specifically, my intuition is that the first two implicatures in both examples could be false, but only if there's a falsifying instance for the indefinite that doesn't overlap with the verifying instance for the corresponding indefinite in the asserted sentence. For example, (13) would be acceptable in a scenario where also the father of some other boys knew all of the answers. At present, I have no account of this intuition, but I would like to come back to this issue in some future research.

8. These two entailments are exactly the implicatures that the first and the second conjunct of (17) have, when they occur as single sentences.

9. In footnote 4, I pointed out that Hirschberg's (1985) proposal runs into exactly the problem observed in the text here.

10. For reasons I don't fully understand disjunction behaves differently from cardinals in the scope of an epistemic modal. Consider (i), which seems to me to 
have the implicature that Kai never did both singing and dancing. This might argue that that the generalization in the text is oversimpliefied:

(i) a. Kai might have (either) sung or danced today.

b. No, Kai might have sung and danced.

11. Of course, it would be possible to stipulate some assumption that does nothing but block implicatures from the scope of an epistemic modal; for example, one might assume that any speaker, who asserts might $\phi$ implies thereby that, for all scalar alternatives $\psi$ of $\phi$ "might $\psi$ and might not $\psi$ ".

12. It follows from the Gricean maxim of brevity that $\mathrm{L}$ and $\mathrm{R}$ can never be used in an assertion.

13. The following discussion is framed in terms of impersonal certainty of a certain proposition, rather than whether the speaker is certain or not of some proposition as assumed e.g. by Gamut (1991). The difference, however, is only in the notation-I want to stick with the impersonal epistemic K-operator that Gazdar (1979) uses.

14. I haven't investigated how cases where a proper subset of all maxims gives rise to a primary implicature are to be treated. Maybe this can force the cancellation of primary implicatures that are drawn from all the maxims. Or, maybe the hearer makes really just one assumption about the speaker. Namely, that he is cooperative, which is equivalent to the conjunction of all of Grice's maxims.

15. Gazdar (1979:136) argues that if a clausal implicature conflicts with a scalar implicature the latter is cancelled. This is reminiscent of the hierarchy between primary and secondary implicatures I proposed. A difference might be, though, that the hierarchy assumed here can be derived in the way shown above.

\section{References}

Atlas, Jay D., and Stephen C. Levinson. 1981. It-clefts, informativeness, and logical form: Radical pragmatics (revised standard version). In Radical Pragmatics, ed. by P. Cole, 1-61. New York: Academic Press.

Beck, Sigrid, and Uli Sauerland. 2001. Cumulativity is needed: A reply to Winter (2000). Natural Language Semantics 9. (to appear).

Chierchia, Gennaro. 2001. Scalar implicatures, polarity phenomena, and the syntax/pragmatics interface. Manuscript, University of Milan.

Gamut, L.T.F. 1991. Logic, Language, and Meaning, Volume 1. Chicago, Illinois: University of Chicago Press.

Gazdar, Gerald. 1979. Pragmatics: Implicature, Presupposition, and Logical Form. New York: Academic Press.

Grice, Paul. 1978. Logic and conversation. In Speech Acts, ed. by P. Cole and J. L. Morgan. New York: Academic Press. (Manuscript of a William James Lecture, 1967).

Hintikka, Jaakko. 1962. Knowledge and Belief. Ithaca, N.Y.: Cornell University Press. 
Hirschberg, Julia. 1985. A Theory of Scalar Implicature. Ph.D. dissertation, University of Pennsylvania, Philadelphia, Penn.

Horn, Laurence R. 1972. On the Semantic Properties of Logical Operators in English. Ph.D. dissertation, University of California, Los Angeles.

— 1989. A Natural History of Negation. Chicago, Ill.: University of Chicago Press.

Levinson, Stephen C. 1983. Pragmatics. Cambridge, UK: Cambridge University Press.

2000. Presumptive Meanings. Cambridge, Mass.: MIT Press.

Matsumoto, Yo. 1995. The conversational condition on Horn scales. Linguistics and Philosophy 18.21-60.

McCawley, James D. 1993. Everything that Linguists have Always Wanted to Know about Logic. Chicago, Ill.: University of Chicago Press.

Scha, Remko. 1984. Distributive, collective, and cumulative quantification. In Truth, Interpretation, and Information, GRASS 2, ed. by J. Groenendijk, M. Stokhof, and T. Janssen, 131-158. Dordrecht, Netherlands, Foris.

Simons, Mandy. 1998. "OR": Issues in the Semantics and Pragmatics of Disjunction. Ph.D. dissertation, Cornell University, Ithaca, N.Y.

Soames, Scott. 1982. How presuppositions are inherited: A solution to the projection problem. Linguistic Inquiry 13.483-545.

von Fintel, Kai, and Irene Heim. 2000. Pragmatics in linguistic theory. Unpublished Classnotes, MIT. 